\title{
UVA/Riboflavin-Induced Apoptosis in Mouse Cornea
}

\author{
F. Wang \\ Department of Ophthalmology, University Hospital Carl Gustav Carus, Dresden University of Technology, \\ Dresden, Germany
}

\section{Key Words}

Ultraviolet irradiation - Riboflavin pretreatment •

Antigen-presenting cells $\cdot$ Cornea $\cdot$ Apoptosis

\begin{abstract}
Background: A mouse model of combined UVA/riboflavin irradiation to eliminate stromal cells and other antigen-presenting cells in the cornea provides the basis for a probably low risk of corneal transplantation. Methods: After abrasion of the epithelium, the central corneas of mouse eyes were treated with UVA/riboflavin in vitro. Histological studies of hematoxylin-eosin and immunohistochemical staining with caspase 3 were performed. Dissected mouse corneas were analyzed by Western blot. Results: Apoptotic cells were shown on the central corneal stroma; a cell-free zone was displayed in the cornea. Numbers of dead cells increased according to cultivation time. However, the endothelium survived due to the adjustment of the irradiation dose. Conclusions: A cell-free zone in the stroma of the mouse cornea was produced by UVA/riboflavin irradiation in vitro. The technique makes possible to prevent or reduce immunological reactions and the risk of graft rejection by pretreatment of the donor cornea, ultimately prolonging graft survival.

Copyright $\odot 2008$ S. Karger AG, Basel
\end{abstract}

\section{Introduction}

Combined UVA irradiation/riboflavin treatment represents a new method, which increases the biomechanical rigidity of the cornea by collagen cross-linking [1]. In clinical trials, the progression of keratoconus could be stopped [2-4]. Possible cytotoxic effects of this procedure on the corneal endothelium have been investigated in in vitro studies and in rabbits $[5,6]$. In keratoplasty, transplant rejection may also be caused by the cells in the donor cornea [7]. The cells are antigen-presenting cells but also keratocytes which are the carriers of immune complexes [8]. Therefore we sought to address in a mouse model the fact that, following collagen cross-linking during UVA/riboflavin irradiation, the stromal cells and especially the antigen-presenting cells might both be removed, which would result in an abolished or at least diminished immunological reaction after keratoplasty. It is thereby important to ensure survival of the nonproliferating or postmitotic corneal endothelial cell layer.

\section{Material and Methods}

In all experiments, 3-month-old female $\mathrm{C} 3 \mathrm{H}$ mice weighing 20-25 g were obtained from the Experimental Center Department (University Hospital Carl Gustav Carus, Dresden University of Technology). All protocols were approved by the Animal Care and Use Committee, and all animals were treated according

Dr. Fan Wang

Department of Ophthalmology, Johann Wolfgang Goethe University Hospital Theodor-Stern-Kai 7

DE-60590 Frankfurt am Main (Germany)

Tel. +49 696301 5056, Fax +49 696301 6586, E-Mail fanwang_hh@yahoo.com
Fax +41 613061234 
to the ARVO Statement for the Use of Animals in Ophthalmic and Vision Research. Seventy-four mouse eyes were utilized for UVA/ riboflavin irradiation. Fifteen eyes without irradiation were investigated as control.

\section{In vitro UV Irradiation}

Enucleated mouse eyes were irradiated with UVA $370 \mathrm{~nm}$ using a single UVA diode (Roithner Lasertechnik, Vienna, Austria) at a distance of $1 \mathrm{~cm}$ from the cornea. The UVA diode was covered with a Cliplite (Conrad Electronics) to get a restricted irradiation area of $2 \mathrm{~mm}$ diameter on the center of the cornea. For abrasion of the corneal epithelium, riboflavin photosensitizer solution containing riboflavin-5-phosphate and dextran T-500 was applied onto the cornea $5 \mathrm{~min}$ before the irradiation and every $5 \mathrm{~min}$ during the 30-min irradiation process (fig. 1a). Different irradiation parameters, involving $0.1-0.5 \%$ riboflavin, $0-20 \%$ dextran and irradiation energy of $0.5-4 \mathrm{~mW} / \mathrm{cm}^{2}$, with an irradiation time of 15-30 min, were regulated in order to get an optimal irradiation condition. The mouse eyes were cultivated in culture medium II (Gibco Invitrogen), consisting of $2.2 \mathrm{~g} / 1 \mathrm{H}_{2} \mathrm{CO}_{3}$, L-glutamine, $12.5 \mathrm{~mm}$ HEPES, penicillin-streptomycin $50 \mathrm{IU} / \mathrm{ml}$ and dextran T-500 (Biochirom AG, Germany), incubated at $37^{\circ} \mathrm{C}$ for 1,2 or 3 days, respectively.

\section{Histological Studies}

Mouse eyes were fixed in $4 \%$ formalin and prepared for paraffin sectioning. The sections were stained with hematoxylin-eosin. Serial sections were immunohistochemically stained using the avidin-biotin-peroxidase complex procedure (Vector Laboratories, Burlingame, Calif., USA) to determine the presence of caspase 3. Briefly, deparaffinized sections were boiled in $10 \mathrm{~mm}$ sodium citrate buffer at $\mathrm{pH} 6.0$ for $10 \mathrm{~min}$ and incubated with diluted blocking solution (1:100) for $1 \mathrm{~h}$. All sections were incubated with diluted primary antibody of anti-rabbit caspase 3 (1:200, Cell Signaling) overnight at $4^{\circ} \mathrm{C}$, with biotinylated secondary goat anti-rabbit antibody (1:100, Vector Laboratories) for $30 \mathrm{~min}$ at room temperature, and with avidin-peroxidase conjugate for $30 \mathrm{~min}$. Sections were developed in $0.05 \%$ diaminobenzidine solution and counterstained with hematoxylin. Negative control incubation was omitted with the primary antibody. All sections were viewed with a Zeiss Axiophot photomicroscope (Germany).

\section{Western Blot Analysis for Cleaved Caspase 3}

Lysates of corneal tissues were prepared by mechanically dissecting intact corneas with a blade and homogenized in lysis buffer (20 mM Tris, pH 7.5, $150 \mathrm{~mm} \mathrm{NaCl}, 1 \%$ Triton X-100, protease cocktail inhibitor, $0.1 \mu \mathrm{g}$ leupeptin, $2 \mu \mathrm{g} / \mathrm{ml}$ aprotinin); homogenates were centrifuged for $10 \mathrm{~min}$, at $10,000 \mathrm{~g}$. Samples were loaded onto $12 \%$ gel and separated by electrophoresis. The proteins were transferred to polyvinyl difluoride membranes (Millipore Corp., USA) by electrophoresis in transfer buffer. Membranes were blocked in blocking buffer of 5\% nonfat dry milk in $0.3 \%$ Tween-20 PBS solution, then incubated in anti-rabbit caspase 3 of 1:100 (Cell Signaling) with blocking buffer overnight at $4{ }^{\circ} \mathrm{C}$. Following incubation in horseradish-peroxidaseconjugated goat anti-rabbit IgG (Jackson Immunoresearch Laboratories) in blocking buffer for $30 \mathrm{~min}$, horseradish peroxidase labeling was determined by enhanced chemiluminescence (Amersham Biosciences, UK), and the membrane was exposed to
X-ray film (Amersham Biosciences). In vitro cultured human retinal pigment epithelial cells that had been treated with staurosporine $(1 \mu \mathrm{g} / \mathrm{ml})$ overnight were used as positive control for cleaved caspase 3 ; nontreated normal cornea tissue was regarded as negative control.

\section{Results}

\section{In vitro UVA Irradiation}

Concerning the in vitro model, the optimal cultivation conditions were: $0.5 \%$ riboflavin, $10 \%$ dextran, irradiation time set to $30 \mathrm{~min}$; following the variable cultivation time from 1 to 3 days, the cell nucleus or nuclear fragment numbers in corneal stroma were reduced. Immunohistochemical staining of caspase-3-positive cells was increased in the stroma from posttreatment day 1 to day 2 on the center and periphery of the cornea. Extensive caspase 3 positive staining was displayed on day 2 after UVA irradiation (fig. 1e). Until day 3 , with the irradiation dose of $1.2 \mathrm{~mW} / \mathrm{cm}^{2}$, almost all of the corneal component cells in the stroma underwent apoptosis, and an absolutely cell-free zone was achieved from the peripheral to the central cornea, except for the endothelium preserved on Descemet's membrane in extenso. However, epithelial cells were still absent in the center of the mouse cornea (fig. 1c). In contrast, when the irradiation doses were increased above $1.2 \mathrm{~mW} / \mathrm{cm}^{2}$, including the keratocytes in the stroma, even the corneal endothelial cells were destroyed and only corneal collagen remained.

\section{Expression of Cleaved Caspase 3 in Irradiated Corneas}

To prove the immunohistological results, UVA-irradiated mouse corneas incubated for 1 or 2 days and normal nontreated corneas were subjected to SDS-PAGE and Western blotting for cleaved caspase 3 expression. A polyclonal antibody that identifies both 17- and 19$\mathrm{kDa}$ subunits of mouse cleaved caspase 3 and human cleaved caspase 3 was used. A faint band of $17 \mathrm{kDa}$ was found on posttreatment day 1 . Another strong band of both 17 and $19 \mathrm{kDa}$ was detected on posttreatment day 2. There was no signal showing on nontreated normal mouse corneas. The apoptotic process developed after UVA irradiation on day 1 , then amounts of apoptotic nuclear cells increased on posttreatment day 2 . The results confirmed the immunohistochemical assay findings that UVA-irradiated corneas contain apoptotic cells (fig. 1f). 


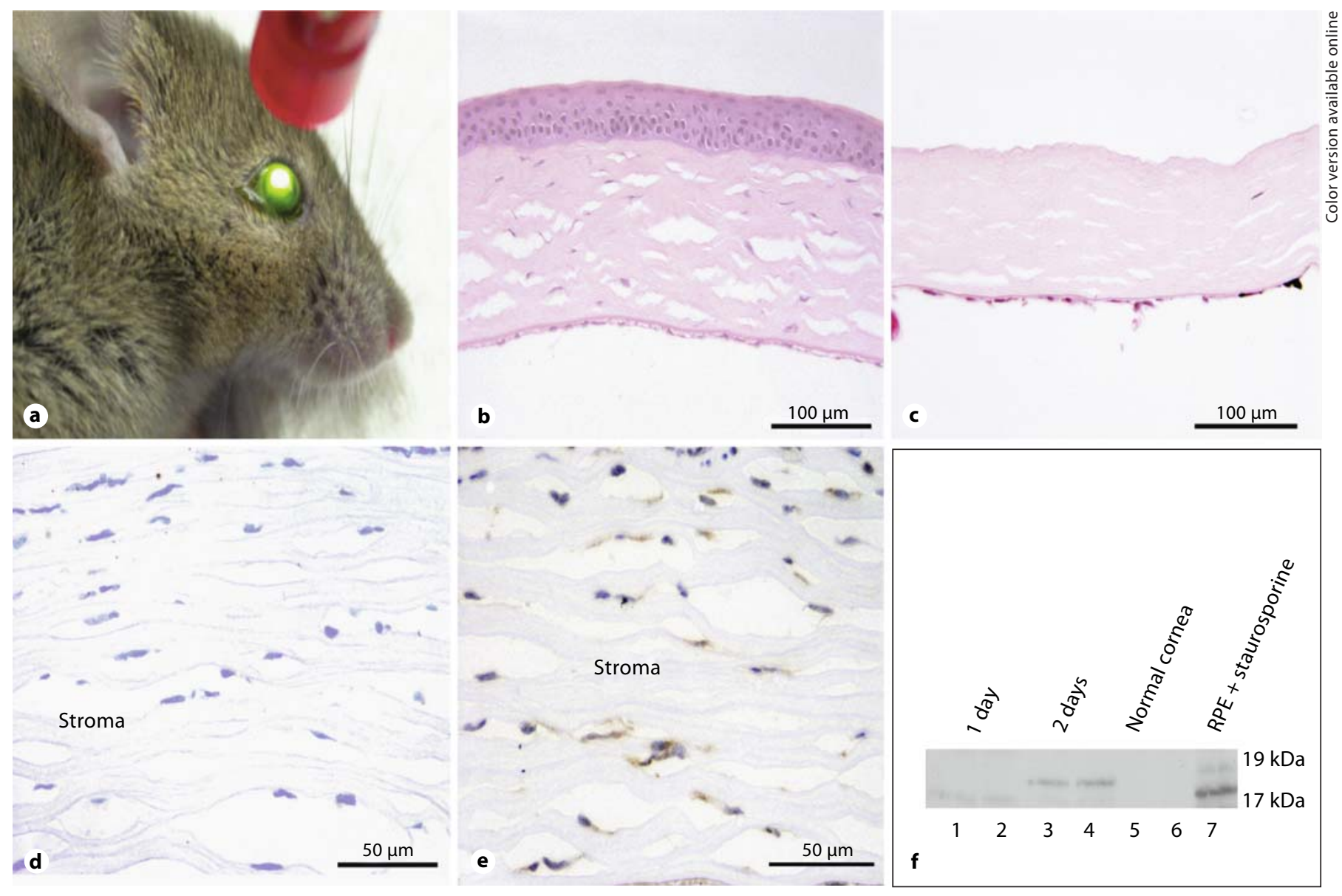

Fig. 1. a UVA/riboflavin irradiation on the mouse eye. The mouse eyes were irradiated with a UVA diode at a distance of $1 \mathrm{~cm}$ from the central cornea. After abrasion of the corneal epithelium, riboflavin photosensitizer solution was dropped onto the cornea 5 min before irradiation and every 5 min during the 30 -min irradiation process. $\mathbf{b}$ Hematoxylin-eosin staining of normal nontreated mouse cornea. $\times 200$. c Hematoxylin-eosin staining of cornea cultivated for 3 days after UVA irradiation. An absolutely cell-free zone was achieved from the peripheral to the central cornea, except for the endothelium preserved on Descemet's mem-

\section{Discussion}

To improve high-risk corneal grafting, research is accomplished to find new therapies, which are more efficient than that used universally. A new and possibly additional concept is to manipulate the antigen-presenting cells in the cornea in order to modulate the immunological state of the transplant [9-12]. Antigen-presenting cells reside in the deeper stromal layers. Numerous dendritic cells reside especially in the anterior stroma [13].

UVA/Riboflavin-Induced Apoptosis in Mouse Cornea brane in extenso. $\times 200$. d Negative control of immunohistochemical staining with caspase 3. e Localization of apoptotic stromal cells irradiated by UVA for 2 days. Caspase-3-positive cells were diffused in the corneal stroma. $\times 400$. $\mathbf{f}$ Western blot analysis of cleaved caspase 3 expression on mouse corneas after UVA/ irradiation cultivated for 1 day (lanes 1 and 2) or 2 days (lanes 3 and 4) and normal nontreated corneas (lanes 5 and 6); lane 7: human retinal pigment epithelial cells (RPE) served as positive control.

Hence we suggested to utilize a physical method in which the cells could be reduced or completely removed, and immunological cells were locally destroyed, also by mechanical abrasion of corneal epithelial cells, with enrichment of antigen-presenting cells. In the present study, a well-established mouse model of UVA irradiation combined with riboflavin was used to induce apoptosis in the corneal stroma in vitro. The process is similar to the human donor cornea cultivation system. The cytotoxic irradiance threshold of $1.2 \mathrm{~mW} / \mathrm{cm}^{2}$ for keratocytes could be confirmed in the cultivated mouse cornea. Based on 
the Lambert-Beer law, the safe threshold for corresponding UVA irradiation on the cultivated human cornea could also be deduced.

In the case of corneal epithelial debridement wounds, the keratocytes beneath the basement membrane undergo apoptosis immediately. Shortly after death, the cells are replaced by new keratocytes by mitosis of adjacent cells, and consequently no further keratocyte response occurs. Approximately $6 \mathrm{~h}$ after injury, the activated keratocytes enter into the cell cycle and subsequently migrate to the site of injury [14]. If the UVA-irradiated corneas are transplanted as donor, based on the above theory, the keratocytes of the recipient could be regenerated from the recipient's edge of the wound bed to the graft. Also the scraped epithelium could be replaced by recipient corneal epithelial cells. Then the donor graft is not recognized as foreign body any more. In principle, the risk of corneal transplant rejection could be reduced or prevented.
In conclusion, UVA/riboflavin treatment significantly promotes apoptosis of mouse corneal stromal cells. The discovery provides the possibility to prevent or reduce immunological reactions and the risk of graft rejection by pretreatment. It supports the further application of this new method in clinical corneal transplantation. Nowadays, an animal model of keratoplasty for preclinical study is under way; many more results need to be investigated.

\section{Acknowledgements}

The author gratefully acknowledges Prof. Richard Funk, Prof. Katrin Engelmann and Prof. Eberhard Spörl. The author thanks the Anatomy and Experiment Center Department for their help.

\section{References}

1 Spoerl E, Huhle M, Seiler T: Induction of cross-links in corneal tissue. Exp Eye Res 1998;66:97-103.

2 Caporossi A, Baiocchi S, Mazzotta C, Traversi C, Caporossi T: Parasurgical therapy for keratoconus by riboflavin-ultraviolet type A rays induced cross-linking of corneal collagen: preliminary refractive results in an Italian study. J Cataract Refract Surg 2006; 32:837-845.

3 Unger G, Schilde T, Spoerl E, Kohlhaas M. Pillunat L: Evaluation of the stiffening effect of riboflavin/UVA treatment in different depth of the cornea. Invest Ophthalmol Vis Sci 2004;45:E-abstract 2888.

4 Wollensak G, Spoerl E, Seiler T: Treatment of keratoconus using riboflavin/UVA-induced crosslinking of the cornea. Am J Ophthalmol 2003;135:620-627.
5 Wollensak G, Spoerl E, Reber F, Pillunat L, Funk R: Corneal endothelial cytotoxicity of riboflavin/UVA treatment in vitro. Ophthalmic Res 2003;35:324-328.

6 Wollensak G, Spoerl E, Wilsch M, Seiler T: Endothelial cell damage after riboflavin-ultraviolet-A treatment in the rabbit. J Cataract Refract Surg 2003;29:1786-1790.

7 Hill JC, Sarvan J, Maske R, Els WJ: Evidence that UV-B irradiation decreases corneal Langerhans cells and improves corneal graft survival in the rabbit. Transplantation 1994; 57:1281-1284.

8 Wachtlin J, Khaireddin R, Hoffmann F: Gewebetypisierung bei der perforierenden Keratoplastik. Ophthalmologe 2003;100: 1021-1030.

9 Moller-Pedersen T, Gavanagh HD, Petroll WM, Jester JV: Neutralizing antibody to TGFbeta modulates stromal fibrosis but not regeneration of photoablative effect following PRK. Curr Eye Res 1998;17:736-747.
10 Nakamura K, Kurosaka D, Bissen-Miyajima $\mathrm{H}$, Tsubota $\mathrm{K}$ : Intact corneal epithelium is essential for the prevention of stromal haze after laser assisted in situ keratomileusis. $\mathrm{Br}$ J Ophthalmol 2001;85:209-213.

11 Hart DN: Dendritic cells: unique leukocyte populations which control the primary immune response. Blood 1997;90:3245-3287.

12 Sprent J: Antigen-presenting cells: professionals and amateurs. Curr Biol 1995;5: 1095-1097.

13 Hamrah P, Liu Y, Zhang Q, Dana MR: The corneal stroma is endowed with a significant number of resident dendritic cells. Invest Ophthalmol Vis Sci 2003;44:581-589.

14 Yamada J, Maruyama K, Sano Y, Kinoshita S, Murata Y, Hamuro J: Promotion of corneal allograft survival by the induction of oxidative macrophages. Invest Ophthalmol Vis Sci 2004;45:448-454. 